\title{
Construction of a linkage map and QTL mapping for fiber quality traits in upland cotton (Gossypium hirsutum $\mathbf{L}$.)
}

\author{
LIANG QingZhi ${ }^{\dagger}$, HU Cheng, HUA Hua, LI ZhaoHu \& HUA JinPing ${ }^{*}$ \\ Department of Plant Genetics and Breeding, College of Agronomy and Biotechnology/Key Laboratory of Crop Heterosis and Utilization of \\ Ministry of Education/Beijing Key Laboratory of Crop Genetic Improvement, China Agricultural University, Beijing 100193, China
}

Received January 21, 2013; accepted February 21, 2013; published online May 31, 2013

\begin{abstract}
With the development in spinning technology, the improvement of cotton fiber quality is becoming more and more important. The main objective of this research was to construct a high-density genetic linkage map to facilitate marker assisted selection for fiber quality traits in upland cotton (Gossypium hirsutum L.). A genetic linkage map comprising 421 loci and covering $3814.3 \mathrm{cM}$, accounting for approximately $73.35 \%$ of the cotton genome, was constructed using an $\mathrm{F}_{2}$ population derived from cross GX1135 $\left(\mathrm{P}_{1}\right) \times$ GX100-2 $\left(\mathrm{P}_{2}\right)$. Forty-four of 49 linkage groups were assigned to the 26 chromosomes. Fiber quality traits were investigated in $F_{2}$ population sampled from individuals, and in $F_{2: 3}$, and $F_{2: 4}$ generations sampled by lines from two sites and one respectively, and each followed a randomized complete block design with two replications. Thirty-nine quantitative trait loci were detected for five fiber quality traits with data from single environments (separate analysis each): 12 for fiber length, five for fiber uniformity, nine for fiber strength, seven for fiber elongation, and six for fiber micronaire, whereas 15 QTLs were found in combined analysis (data from means of different environments in $\mathrm{F}_{2: 3}$ generation). Among these QTLs, $q F L$-chr5-2 and $q F L$-chrl4-2 for fiber length were detected simultaneously in three generations (four environments) and verified further by combined analysis, and these QTLs should be useful for marker assisted selection to improve fiber quality in upland cotton.
\end{abstract}

upland cotton (Gossypium hirsutum L.), fiber quality traits, genetic linkage map, marker assisted selection, QTLs

Citation: $\quad$ Liang Q Z, Hu C, Hua H, et al. Construction of a linkage map and QTL mapping for fiber quality traits in upland cotton (Gossypium hirsutum L.). Chin Sci Bull, 2013, 58: 3233-3243, doi: 10.1007/s11434-013-5807-1

Cotton (Gossypium spp.) is the most important natural fiber crop and is served as the second largest source of edible oil in the world. The genus Gossypium includes 45 diploid and 5 tetraploid species [1]. Cultivated types include two diploids, Gossypium herbaceum L. (A1) and Gossypium arboreum L. (A2), and two tetraploids, Gossypium hirsutum L. (AD1) and Gossypium barbadense L. (AD2). Of these, upland cotton ( $G$. hirsutum) dominates the production of cotton fiber and accounts for $95 \%$ of the world's total production of cotton [2].

Cotton fiber is widely used as the raw materials for the textile industry. With the changes in spinning technology and diversificated uses, the improvement of cotton fiber

$\dagger$ Present address: Xiangyang Academy of Agricultural Sciences, Xiangyang 441057, China *Corresponding author (email: jinping_hua@ @au.edu.cn) quality is becoming extremely important [3]. However, fiber quality has a negative genetic correlation with lint yield [4,5], which has long been a major problem in cotton breeding. Recently, Chen et al. [6] integrated genome-wide expression profiling markers with linkage analysis to reveal the molecular mechanisms underlying fiber differential development between $G$. barbadense and G. hirsutum. The results suggested that differential gene regulation causes the difference in the quality of fiber between $G$. barbadense and G. hirsutum.

The development of molecular markers has made it possible for plant breeders to find a rapid and precise alternative approach for improving cotton lint yield and fiber quality traits [7]. Quantitative trait loci (QTL) mapping of fiber quality traits can be very helpful in revealing the genetic basis of various fiber quality characteristics and providing 
important information for improving cotton breeding strategies. QTLs conferring fiber quality traits have been identified and mapped using molecular markers in interspecific populations from crosses between G. hirsutum and G. barbadense [8-10]. A high-density interspecific genetic map was constructed, which includes 2316 loci on the 26 cotton chromosomes [11]. Unfortunately, these genetic maps developed from interspecific hybridization currently have limited use in conventional breeding [12]. Instead, saturated intraspecific upland cotton maps need to be constructed to offer more useful information. However, most intraspecific genetic maps were characterized by low marker coverage in the genome because the degree of molecular marker polymorphism is relatively low within G. hirsutum. Apparently, the current upland cotton genetic maps cannot meet the required coverage necessary for marker assisted selection (MAS). Therefore, it is necessary to construct a high-density genetic map of upland cotton using a large number of molecular markers [13].

To improve our understanding of the structure and genetic variability of the G. hirsutum genome, a comprehensive PCR-based marker linkage map for fiber quality that covered $70.6 \%$ of the upland cotton genome was constructed [14]. More recently, Zhang et al. [15] developed a composite crossing population in upland cotton and constructed a genetic map spanned $4184.4 \mathrm{~cm}$, covering approximately $94.1 \%$ of the entire tetraploid cotton genome and containing 978 simple sequence repeat (SSR) loci. A draft physical map of a D-genome cotton species (Gossypium raimondii Ulbr., D5) has been completed [16-18] and the G. hirsutum genome is being sequenced (http://www.monsanto.com/newsviews/Pages/Monsanto-Illumina-Key-Milestone-Cotton-Ge nome-Sequencing.aspx), which will provide rich SSR markers and functional markers for construction of high-density genetic linkage map and QTL mapping for fiber quality traits to facilitate MAS. In the present study, a high-density genetic map was constructed using an $F_{2}$ population derived from an upland cotton hybrid and used for tagging cotton fiber quality in upland cotton.

\section{Materials and methods}

\subsection{Plant materials}

The hybrid of upland cotton 'Xinza 1' (G. hirsutum) from the cross of GX1135 (G. hirsutum) $\left(\mathrm{P}_{1}\right)$ and GX100-2 $(G$. hirsutum) $\left(\mathrm{P}_{2}\right)$ with significant competitive heterosis was bred by the Guoxin Seed Company (Hebei Province, China), and the hybrid was released as a cultivar in Anhui Province in 2006. In the present study, $F_{1}$ seeds were developed from a manual emasculated cross between $P_{1}$ and $P_{2}$ grown at the Guoxin South Propagation Station in Sanya, Hainan Province in winter 2006, and the $F_{1}$ seeds were then grown at the Xinzhou Cotton Breeding Station (Wuhan, 30 $0^{\circ} 34^{\prime} \mathrm{N}, 14^{\circ} 16^{\prime} \mathrm{E}$ ) in April 2007. The genotype of the $F_{1}$ individuals was dis- tinguished by a codominant molecular marker, and an $F_{1}$ individual was self-pollinated to produce $\mathrm{F}_{2}$ seeds. A total of 256 randomly selected $F_{2}$ seeds were cultured in nutrient solution [19] in green house at the China Agricultural University (Beijing) in October 2007. Each $\mathrm{F}_{2}$ seedling was tagged, and root traits were scanned to survey the characters and development of relative traits (the results will be reported in another paper). All the $\mathrm{F}_{2}$ seedlings were transported by air and transplanted at the Guoxin South Propagation Station and self-pollinated to produce $\mathrm{F}_{3}$ seeds. The $\mathrm{F}_{3}$ family lines were bulk self-pollinated to produce $\mathrm{F}_{4}$ seeds. A population of $173 \mathrm{~F}_{2: 3}$ family lines were planted with the parents and $F_{1}$ as controls in 2008, and a population of 173 $\mathrm{F}_{2: 4}$ family lines were planted with the parents and $\mathrm{F}_{2}$ as controls in 2009.

\subsection{Field planting and examination}

The field planting followed a randomized complete block design with two replications, at the Quzhou Experimental Station of the China Agricultural University (Handan, $36^{\circ} 78^{\prime} \mathrm{N}$, $\left.114^{\circ} 92^{\prime} \mathrm{E}\right)$ and the Guoxin Cotton Breeding Experimental Station (Cangzhou, $38^{\circ} 43^{\prime} \mathrm{N}, 116^{\circ} 09^{\prime} \mathrm{E}$ ). The nutrition bowl cultivation method was implemented, and 25-day-old seedlings were transplanted in spaced $30 \mathrm{~cm}$ between plants in two-row plot. Plots were $4 \mathrm{~m}$ in length with $80 \mathrm{~cm}$ row spacing for the experiment at Handan, and $4 \mathrm{~m}$ in length and $80 \mathrm{~cm}, 60 \mathrm{~cm}$ row spacing alternately for the experiment at Cangzhou in 2008. The direct seeding method was carried out and planted in two-row plot, $80 \mathrm{~cm}, 60 \mathrm{~cm}$ wide alternately and $4 \mathrm{~m}$ long in Handan in 2009. Field management followed conventional standard field practices.

Fiber samples were collected from plants growing in the middle of the interior of each plot. Fiber quality traits were investigated in $\mathrm{F}_{2}$ population sampled from individuals, and in $F_{2: 3}$, and $F_{2: 4}$ generations sampled by lines from two sites and one, respectively, and each followed a randomized complete block design with two replications. Fiber quality traits were measured with an HVI 900 instrument (USTER $^{\circledR}$ HVISPECTRUM, SPINLAB, United States) at the Cotton Fiber Quality Inspection and Test Center of Ministry of Agriculture (Anyang, China). The fiber quality traits included $2.5 \%$ fiber span length (mm), fiber length uniformity ratio, fiber strength (cN/tex), fiber elongation, and fiber fineness (micronaire reading).

\subsection{DNA extraction and genotype analysis}

Young leaves were collected from labeled $\mathrm{F}_{2}, \mathrm{P}_{1}, \mathrm{P}_{2}$, and $\mathrm{F}_{1}$ individuals, frozen in liquid nitrogen, and stored at $-80^{\circ} \mathrm{C}$ until analysis. Genomic DNA was individually extracted according to the CTAB method [20]. A total of 16405 SSR primer pairs were used to screen for polymorphic markers between $\mathrm{P}_{1}$ and $\mathrm{P}_{2}$. Among these primers, 13468 pairs of primers included types of BNL, NAU, TM, JESPER, CIR, 
HAU, CM, MUSS, MUSB, and MUCS, which were previously described in detail [8-10,14,21,22]; information on these primers can be obtained from the Cotton Microsatellite Database (http://www.cottonmarker.org). The remaining 2937 primer pairs were designed and developed from the DNA sequence library [23]. The 450 SSR primer pairs that showed polymorphisms between the two mapping parents were used to genotype 173 individuals from the $F_{2}$ population. The procedure for SSR analysis was that described by Mei et al. [24].

\subsection{Map construction and QTL analysis}

MAPMAKER 3.0b [25] was implemented to construct a genetic linkage map. The assignment of linkage groups to chromosomes was based on backbone linkage maps $[4,8,9$, $13,14,26]$. When no chromosome inference was available, the linkage group was described as un $\times x$, where $x \times$ refers to its serial number. QTLs were analyzed by composite interval mapping [27] using the computer program QTL Cartographer 2.5 [28].

A stringent LOD threshold of 3.0 was used to declare a suggestive QTL, as described by Lander et al. [29], whereas the same QTL in another environment with LOD of at least 2.0 was considered to be a common QTL, as described by Shen et al. [30]. QTL mapping was carried out for data sets from single environment (separate analysis for each environment in three generations) following Zhang and $\mathrm{Xu}$ [31], and a set of data from the means of different environments in $\mathrm{F}_{2: 3}$ generation (combined analysis). The graphic representation of the linkage group and QTLs marked were created by Map Chart 2.2, following Voorrips et al. [32].

\section{Results}

\subsection{Evaluation of fiber quality traits in the $F_{2}, F_{2: 3}, F_{2: 4}$ populations and two parents}

Midparent heterosis of fiber quality traits was assessed. 'Xinza 1' was an elite hybrid upland cotton and showed significant heterosis in seed-cotton yield and lint yield, the mid-parent heterosis values were $56.23 \%$ and $62.01 \%$, respectively. In contrast, fiber quality traits showed almost no heterosis. The phenotypic data for fiber quality traits of the $\mathrm{F}_{2}, \mathrm{~F}_{2: 3}, \mathrm{~F}_{2: 4}$ populations and $\mathrm{F}_{1}$, the two parents are summarized in Table 1 . The values of most fiber quality traits in the $F_{2}, F_{2: 3}$ and $F_{2: 4}$ populations fell between those of the two parents. Skewness and kurtosis values (data not shown) were calculated; the results indicated that all fiber quality traits fit a normal distribution and all the traits expressed transgressive segregation in both directions in the $F_{2}, F_{2: 3}$ and $\mathrm{F}_{2: 4}$ populations (Figure $\mathrm{S} 1$ ).

Table 1 Phenotypic values for fiber quality traits of the $F_{2}, F_{2: 3}$, and $F_{2: 4}$ populations and midparent heterosis of $F_{1}$ and $F_{2}$

\begin{tabular}{|c|c|c|c|c|c|c|c|c|}
\hline Traits & Mean & $\mathrm{SD}$ & Min & $\operatorname{Max}$ & GX1135 & GX100-2 & Xinza 1 & $\begin{array}{c}\text { Midparent } \\
\text { heterosis }(\%)\end{array}$ \\
\hline \multicolumn{9}{|l|}{$07 \mathrm{Hn}^{\mathrm{a})}$} \\
\hline Fiber length (mm) & 28.31 & 0.94 & 25.65 & 31.65 & - & - & - & - \\
\hline Fiber uniformity ratio & 83.34 & 1.18 & 79.90 & 85.80 & - & - & - & - \\
\hline Fiber strength (cN/tex) & 25.95 & 1.33 & 22.00 & 29.70 & - & - & - & - \\
\hline Fiber elongation & 6.36 & 0.14 & 5.80 & 6.70 & - & - & - & - \\
\hline Micronaire & 3.65 & 0.51 & 2.16 & 4.92 & - & - & - & - \\
\hline $08 \mathrm{Q} z^{\mathrm{b})}$ & & & & & & & $\mathrm{F}_{1}$ & \\
\hline Fiber length (mm) & 29.72 & 0.88 & 27.01 & 31.88 & 29.88 & 29.51 & 30.17 & 1.60 \\
\hline Fiber uniformity ratio & 84.73 & 0.77 & 82.50 & 86.90 & 84.15 & 84.80 & 83.40 & -1.27 \\
\hline Fiber strength $(\mathrm{cN} / \mathrm{tex})$ & 29.36 & 1.10 & 26.55 & 31.90 & 29.50 & 28.90 & 28.95 & -0.86 \\
\hline Fiber elongation & 6.51 & 0.09 & 6.15 & 6.70 & 6.55 & 6.50 & 6.45 & -1.15 \\
\hline Micronaire & 4.64 & 0.38 & 3.55 & 5.72 & 5.31 & 4.59 & 4.26 & -14.00 \\
\hline $08 \mathrm{Hj}^{\mathrm{c})}$ & & & & & & & $\mathrm{F}_{1}$ & \\
\hline Fiber uniformity ratio & 84.13 & 0.91 & 81.90 & 86.55 & 85.00 & 83.50 & 83.70 & -0.65 \\
\hline Fiber strength (cN/tex) & 28.32 & 1.14 & 25.80 & 31.85 & 29.05 & 27.50 & 28.10 & -0.62 \\
\hline Fiber elongation & 6.42 & 0.11 & 6.10 & 6.70 & 6.40 & 6.35 & 6.25 & -1.96 \\
\hline Micronaire & 4.84 & 0.41 & 3.63 & 6.15 & 4.95 & 4.65 & 4.15 & -13.60 \\
\hline 09Qz $z^{\mathrm{d})}$ & & & & & & & $\mathrm{F}_{2}$ & \\
\hline Fiber length (mm) & 30.21 & 0.87 & 28.14 & 32.59 & 29.09 & 30.11 & 30.62 & 3.45 \\
\hline Fiber uniformity ratio & 84.65 & 0.89 & 82.45 & 86.40 & 84.30 & 85.40 & 85.85 & 1.18 \\
\hline Fiber strength (cN/tex) & 32.82 & 1.23 & 29.75 & 36.20 & 32.50 & 31.50 & 32.15 & 0.47 \\
\hline Fiber elongation & 6.70 & 0.09 & 6.45 & 6.95 & 6.60 & 6.65 & 6.80 & 2.64 \\
\hline Micronaire & 3.91 & 0.30 & 3.12 & 4.66 & 4.09 & 3.85 & 4.61 & 16.14 \\
\hline
\end{tabular}

a) Fiber quality data of $\mathrm{F}_{2}$ harvested from Hainan in 2007 ; b) Fiber quality data of $\mathrm{F}_{2: 3}$ harvested from Handan in 2008 ; c) Fiber quality data of $\mathrm{F}_{2: 3}$ harvested from Cangzhou in 2008; d) Fiber quality data of $\mathrm{F}_{2: 4}$ harvested from Handan in 2009. -, missing data. 


\subsection{Correlation analyses of common fiber quality traits among the $F_{2}, F_{2: 3}$ and $F_{2: 4}$ generations}

Correlation analysis was carried out using the mean values of $F_{2}, F_{2: 3}$ and $F_{2: 4}$ (Table 2). The majority of fiber quality traits were significantly associated with each other. Correlation analysis between traits of different generations was conducted using the mean value of the four environments too (Table 3). All correlation of fiber length was significantly positively correlated among generations, and the correlation coefficients among generations varied greatly from 0.31 to 0.52 . The majority of correlation coefficient of fiber uniformity ratio was significantly positively correlated among generations. Correlation analysis of fiber strength, fiber elongation and micronaire showed the similar tendency among generations.

\subsection{Linkage map construction}

To construct a high-density genetic linkage map in upland

Table 2 Correlation analyses among fiber quality traits of the $\mathrm{F}_{2}, \mathrm{~F}_{2: 3}$, and $\mathrm{F}_{2: 4}$ populations

\begin{tabular}{|c|c|c|c|c|c|}
\hline Trait & Population & Fiber length & Fiber uniformity ratio & Fiber strength & Fiber elongation \\
\hline \multirow[t]{4}{*}{ Fiber uniformity ratio } & $07 \mathrm{Hn} \mathrm{F}_{2}{ }^{\mathrm{a}}$ & $0.51^{* *}$ & & & \\
\hline & $08 \mathrm{Qz} \mathrm{F} \mathrm{F}_{2: 3}^{\mathrm{b})}$ & $0.46^{* *}$ & & & \\
\hline & $08 \mathrm{Hj} \mathrm{F}_{2: 3}^{\mathrm{c})}$ & $0.48^{* * *}$ & & & \\
\hline & 09Qz F $\mathrm{F}_{2: 4}^{\mathrm{d})}$ & $0.46^{* *}$ & & & \\
\hline \multirow[t]{4}{*}{ Fiber strength } & $07 \mathrm{Hn} \mathrm{F}_{2}$ & $0.52^{* *}$ & $0.48^{* *}$ & & \\
\hline & $08 \mathrm{Qz} \mathrm{F}_{2: 3}$ & $0.71^{* *}$ & $0.35^{* *}$ & & \\
\hline & $08 \mathrm{Hj} \mathrm{F}_{2: 3}$ & $0.70^{* * *}$ & $0.49^{* *}$ & & \\
\hline & 09Qz $F_{2: 4}$ & $0.58^{* *}$ & $0.37^{* *}$ & & \\
\hline \multirow[t]{4}{*}{ Fiber elongation } & $07 \mathrm{Hn} \mathrm{F}_{2}$ & $0.45^{* *}$ & $0.41^{* *}$ & $0.79^{* *}$ & \\
\hline & $08 \mathrm{Qz} \mathrm{F}_{2: 3}$ & $0.56^{* *}$ & $0.45^{* *}$ & $0.47^{* *}$ & \\
\hline & $08 \mathrm{Hj} \mathrm{F}_{2: 3}$ & $0.60^{* * *}$ & $0.56^{* *}$ & $0.62^{* *}$ & \\
\hline & 09Qz $\mathrm{F}_{2: 4}$ & $0.53^{* *}$ & $0.33^{* *}$ & $0.60^{* *}$ & \\
\hline \multirow[t]{4}{*}{ Micronaire } & $07 \mathrm{Hn} \mathrm{F}_{2}$ & $-0.28^{* *}$ & 0.11 & 0.09 & $0.35^{* *}$ \\
\hline & 08Qz $\mathrm{F}_{2: 3}$ & -0.12 & $0.27^{* *}$ & $-0.38^{* *}$ & $0.24^{* *}$ \\
\hline & $08 \mathrm{Hj} \mathrm{F}_{2: 3}$ & 0.02 & $0.32^{* *}$ & $-0.18^{*}$ & $0.34^{* *}$ \\
\hline & 09Qz F F:4 & -0.12 & 0.07 & $-0.17^{*}$ & $0.19^{*}$ \\
\hline
\end{tabular}

a) Fiber quality data of $F_{2}$ harvested from Hainan in 2007; b) Fiber quality data of $F_{2: 3}$ harvested from Handan in 2008; c) Fiber quality data of $F_{2: 3}$ harvested from Cangzhou in 2008; d) Fiber quality data of $\mathrm{F}_{2: 4}$ harvested from Handan in 2009. “*” and “**” indicate that the correlation is significant at 0.05 and 0.01 probability levels, respectively.

Table 3 Correlation analyses of the same fiber quality traits among different generations

\begin{tabular}{|c|c|c|c|}
\hline Trait & $07 \mathrm{Hn} \mathrm{F}_{2}{ }^{\mathrm{a})}$ & $08 \mathrm{Qz} \mathrm{F} \mathrm{F}_{2: 3}^{\mathrm{b})}$ & $08 \mathrm{Hj} \mathrm{F}_{2: 3}{ }^{\mathrm{c}}$ \\
\hline 08Qz F $F_{2: 3}$ Fiber length & $0.45^{* *}$ & & \\
\hline $08 \mathrm{Hj} \mathrm{F}_{2: 3}$ Fiber length & $0.40^{* *}$ & $0.52^{* *}$ & \\
\hline 09Qz $\mathrm{F}_{2: 4}{ }^{\mathrm{d})}$ Fiber length & $0.31^{* *}$ & $0.51^{* *}$ & $0.41^{* *}$ \\
\hline $08 \mathrm{Qz} \mathrm{F}_{2: 3}$ Fiber uniformity ratio & $0.16^{*}$ & & \\
\hline $08 \mathrm{Hj} \mathrm{F}_{2: 3}$ Fiber uniformity ratio & 0.05 & $0.26^{* *}$ & \\
\hline $09 \mathrm{Qz} \mathrm{F}_{2: 4}$ Fiber uniformity ratio & $0.20^{* *}$ & $0.24^{* *}$ & $0.17^{*}$ \\
\hline $08 \mathrm{Qz} \mathrm{F}_{2: 3}$ Fiber strength & $0.34^{* *}$ & & \\
\hline $08 \mathrm{Hj} \mathrm{F}_{2: 3}$ Fiber strength & 0.13 & $0.32^{* *}$ & \\
\hline $09 \mathrm{Qz} \mathrm{F}_{2: 4}$ Fiber strength & 0.11 & $0.48^{* * *}$ & $0.35^{* *}$ \\
\hline 08Qz $\mathrm{F}_{2: 3}$ Fiber elongation & $0.24^{* *}$ & & \\
\hline $08 \mathrm{Hj} \mathrm{F}_{2: 3}$ Fiber elongation & -0.12 & $0.19^{*}$ & \\
\hline 09Qz $\mathrm{F}_{2: 4}$ Fiber elongation & 0.08 & $0.32^{* *}$ & $0.32^{* *}$ \\
\hline 08Qz F $F_{2: 3}$ Micronaire & 0.06 & & \\
\hline $08 \mathrm{Hj} \mathrm{F}_{2: 3}$ Micronaire & 0.07 & $0.24^{* *}$ & \\
\hline 09Qz F Fi4 Micronaire & 0.05 & $0.32^{* *}$ & $0.25^{* *}$ \\
\hline
\end{tabular}

a) Fiber quality data of $\mathrm{F}_{2}$ harvested from Hainan in 2007; b) Fiber quality data of $\mathrm{F}_{2: 3}$ harvested from Handan in 2008; c) Fiber quality data of $\mathrm{F}_{2: 3}$ harvested from Cangzhou in 2008; d) Fiber quality data of $\mathrm{F}_{2: 4}$ harvested from Handan in 2009. “*” and “**” indicate that the correlation is significant at 0.05 and 0.01 probability levels, respectively. 
cotton, 16405 primer pairs were screened between $\mathrm{P}_{1}$ and $\mathrm{P}_{2}$. The 450 SSR primer pairs that showed polymorphism between the two mapping parents were used to construct a linkage map using the $\mathrm{F}_{2}$ population. Chi-square goodnessof-fit test was conducted to determine whether the genotypic frequencies differ significantly from the expected segregation ratio. Most of the loci (439 of 450) fitted to the segregation ratio of $1: 2: 1$ or $1: 1$ and were used to construct the linkage map. The genetic linkage map, consisting of 421 loci linked into 49 groups and left 29 loci unlinked and covering $3814.3 \mathrm{~cm}$ of the total recombination length of the cotton genome, was constructed with an average interval of $8.89 \mathrm{cM}$ between adjacent loci and accounted for approxi- mately $73.35 \%$ of the recombination length of the upland cotton genome. Forty-four of 49 linkage groups were assigned to the 26 chromosomes based on previously chromosome-anchored backbone linkage maps [8-10,14,21,22]. The remaining five linkage groups named ' $U n$ 01' to 'Un 05 ' tentatively could not be associated with any chromosome.

\subsection{QTL mapping for fiber quality traits}

Thirty-nine QTLs for fiber quality traits were identified in the four environments in separate analysis, and fifteen of them were detected in the combined analysis in $\mathrm{F}_{2: 3}$ generation (Table 4).

Table 4 QTLs for fiber quality traits in the $\mathrm{F}_{2}, \mathrm{~F}_{2: 3}$, and $\mathrm{F}_{2: 4}$ populations identified using composite interval mapping

\begin{tabular}{|c|c|c|c|c|c|c|c|c|c|c|}
\hline \multirow{2}{*}{$\frac{\text { Trait }}{\text { Fiber length }}$} & \multirow{2}{*}{$\frac{\text { Environment }}{\text { 08Qz }}$} & \multirow{2}{*}{$\frac{\mathrm{QTL}}{q F L-\operatorname{chr} 5-1^{*}}$} & \multirow{2}{*}{$\frac{\text { Chr. }}{5}$} & \multicolumn{2}{|c|}{ Maker interval } & \multirow{2}{*}{$\frac{\text { Position }(\mathrm{cM})}{35.68}$} & \multirow{2}{*}{$\frac{\text { LOD }}{7.46}$} & \multirow{2}{*}{$\frac{\mathrm{A}^{\mathrm{a})}}{-0.52}$} & \multirow{2}{*}{$\frac{\mathrm{D}^{\mathrm{b})}}{-0.01}$} & \multirow{2}{*}{$\frac{\operatorname{Var}(\%)^{\mathrm{c})}}{14.77}$} \\
\hline & & & & MUSS193 & NAU2865 & & & & & \\
\hline & $08 \mathrm{Hj}$ & & 5 & MUSS193 & NAU2865 & 33.68 & 9.45 & -0.62 & -0.08 & 20.12 \\
\hline & 07Hn & $q F L-\operatorname{chr} 5-2 *^{\#}$ & 5 & NAU4034 & HAU1316 & 44.29 & 2.47 & -0.08 & -0.29 & 5.40 \\
\hline & 08Qz & & 5 & HAU1603 & TMB1296 & 38.92 & 7.42 & -0.50 & 0.02 & 14.06 \\
\hline & $08 \mathrm{Hj}$ & & 5 & NAU4034 & HAU1316 & 54.29 & 5.32 & -0.48 & 0.19 & 15.73 \\
\hline & 09Qz & & 5 & HAU1315 & NAU4034 & 42.67 & 7.62 & -0.58 & 0.15 & 17.43 \\
\hline & 08Qz & $q F L-\operatorname{chr} 10-1{ }^{* \#}$ & 10 & BNL2960 & CGR5873 & 79.66 & 4.58 & 0.34 & 0.10 & 10.50 \\
\hline & 09Qz & & 10 & CGR5873 & GH144 & 83.43 & 2.13 & 0.17 & 0.12 & 3.98 \\
\hline & 08Qz & $q F L-c h r 13-1{ }^{* \#}$ & 13 & DPL687 & DPL286 & 140.49 & 8.76 & -0.47 & -0.31 & 20.18 \\
\hline & $08 \mathrm{Hj}$ & & 13 & DPL687 & DPL286 & 140.49 & 3.07 & -0.26 & -0.20 & 6.56 \\
\hline & 07Hn & $q F L-c h r 14-1 *^{*}$ & 14 & HAU1455 & GH120 & 72.60 & 2.77 & 0.37 & -0.14 & 6.51 \\
\hline & $08 \mathrm{Qz}$ & & 14 & CGR6683 & HAU1057 & 83.06 & 3.15 & 0.30 & -0.06 & 5.54 \\
\hline & $08 \mathrm{Hj}$ & & 14 & DPL565 & CGR6683 & 78.05 & 2.58 & 0.34 & -0.15 & 5.37 \\
\hline & 07Hn & $q F L-c h r 14-2 * \#$ & 14 & HAU1057 & NAU3839 & 83.85 & 2.47 & 0.35 & -0.09 & 5.96 \\
\hline & $08 \mathrm{Qz}$ & & 14 & NAU3308 & CGR6802 & 88.52 & 3.42 & 0.33 & -0.05 & 6.27 \\
\hline & $08 \mathrm{Hj}$ & & 14 & CGR6802 & CGR6784 & 91.93 & 3.71 & 0.41 & -0.18 & 7.34 \\
\hline & 09Qz & & 14 & BNL3033 & BNL2469 & 84.44 & 3.25 & 0.35 & -0.13 & 5.98 \\
\hline & 07Hn & $q F L-c h r 3-1$ & 3 & CER028 & GH663 & 12.54 & 3.02 & 0.07 & 0.37 & 6.91 \\
\hline & $08 \mathrm{Hj}$ & $q F L-c h r 3-2$ & 3 & CGR6528 & CGR6017 & 39.95 & 3.56 & 0.39 & -0.14 & 6.33 \\
\hline & 09Qz & $q F L-c h r 8-1$ & 8 & GH398 & DPL090 & 54.13 & 3.17 & -0.15 & 0.47 & 7.41 \\
\hline & 09Qz & $q F L-c h r 11-1$ & 11 & CGR5602 & DPL050a & 69.77 & 4.22 & 0.24 & 0.20 & 10.64 \\
\hline & 07Hn & $q F L-c h r 12-1$ & 12 & DPL303 & NAU943 & 13.49 & 3.77 & -0.41 & 0.01 & 9.24 \\
\hline \multirow{6}{*}{$\begin{array}{l}\text { Fiber uniformity } \\
\text { ratio }\end{array}$} & $08 \mathrm{Hj}$ & & 2 & JESP304 & CIR112 & 34.6 & 3.01 & 0.22 & -0.45 & 6.09 \\
\hline & $08 \mathrm{Hj}$ & $q F U-\operatorname{chr} 5-1{ }^{*}$ & 5 & MUSS193 & NAU2865 & 35.68 & 6.54 & -0.39 & -0.28 & 15.93 \\
\hline & 08Qz & & 5 & NAU2865 & GH388 & 37.34 & 4.46 & -0.41 & 0.02 & 11.42 \\
\hline & 09Qz & $q F U-\operatorname{chr} 14-1$ & 14 & CGR5675 & CGR5871a & 37.57 & 3.17 & 0.00 & 0.40 & 7.12 \\
\hline & 07Hn & $q F U-\operatorname{chr} 18-1$ & 18 & GH60 & DC40150a & 35.85 & 3.38 & 0.32 & 0.35 & 9.11 \\
\hline & $08 \mathrm{Hj}$ & $q F U-\operatorname{chr} 26-1$ & 26 & BNL2495 & DPL070 & 89.09 & 3.52 & 0.44 & 0.09 & 7.75 \\
\hline Fiber & 07Hn & $q F S-\operatorname{chr} 1-1 *$ & 1 & DPL182 & DC40175 & 77.62 & 3.26 & -0.41 & 0.78 & 7.92 \\
\hline
\end{tabular}


(Continued)

\begin{tabular}{|c|c|c|c|c|c|c|c|c|c|c|}
\hline \multirow{2}{*}{$\frac{\text { Trait }}{\text { strength }}$} & \multirow{2}{*}{$\begin{array}{c}\text { Environment } \\
08 \mathrm{Hj}\end{array}$} & \multirow[t]{2}{*}{ QTL } & \multirow{2}{*}{$\begin{array}{c}\text { Chr. } \\
1\end{array}$} & \multicolumn{2}{|c|}{ Maker interval } & \multirow{2}{*}{$\frac{\text { Position }(\mathrm{cM})}{87.83}$} & \multirow{2}{*}{$\begin{array}{l}\text { LOD } \\
2.18\end{array}$} & \multirow{2}{*}{$\begin{array}{c}\mathrm{A}^{\mathrm{a})} \\
-0.19\end{array}$} & \multirow{2}{*}{$\frac{\mathrm{D}^{\mathrm{b})}}{0.58}$} & \multirow{2}{*}{$\frac{\operatorname{Var}(\%)^{\mathrm{c}}}{7.06}$} \\
\hline & & & & DC40175 & CIR307 & & & & & \\
\hline & $09 \mathrm{Qz}$ & & 1 & CIR307 & HAU1417b & 100.44 & 3.47 & -0.48 & 0.85 & 12.46 \\
\hline & 08Qz & $q F S-\operatorname{ch} r 5-1 *^{\#}$ & 5 & NAU2865 & GH388 & 37.34 & 3.39 & -0.43 & -0.04 & 7.35 \\
\hline & $08 \mathrm{Hj}$ & & 5 & MUSS193 & NAU2865 & 35.68 & 4.69 & -0.37 & -0.32 & 11.65 \\
\hline & 09Qz & & 5 & NAU2865 & GH388 & 37.34 & 3.24 & -0.59 & 0.18 & 7.78 \\
\hline & $08 \mathrm{Qz}$ & qFS-chr13-1*\# & 13 & DPL687 & DPL286 & 140.49 & 8.22 & -0.54 & -0.51 & 22.08 \\
\hline & $09 \mathrm{Qz}$ & & 13 & DPL687 & DPL286 & 142.49 & 2.58 & -0.10 & -0.54 & 7.14 \\
\hline & 08Qz & $q F S-\operatorname{chr} 18-1{ }^{* \#}$ & 18 & CIR099 & CIR216 & 67.40 & 2.19 & -0.46 & 0.52 & 7.70 \\
\hline & 09Qz & & 18 & DPL077 & CIR099 & 52.20 & 2.36 & -0.51 & 0.20 & 6.33 \\
\hline & $08 \mathrm{Qz}$ & $q F S$-chr1-2 & 1 & NAU3254 & NAU2343 & 130.31 & 3.64 & -0.49 & 0.06 & 7.60 \\
\hline & 07Hn & $q F S$-chr8-1 & 8 & NAU4045 & CGR6129 & 34.52 & 3.28 & 0.70 & -0.13 & 8.91 \\
\hline & $08 \mathrm{Hj}$ & $q F S$-chr17-1 & 17 & HAU1413a & HAU1417a & 0.01 & 3.61 & 0.33 & -0.04 & 8.45 \\
\hline & 08Qz & $q F S$-chr18-2 & 18 & BNL243 & CER168 & 19.66 & 3.29 & 0.28 & -0.69 & 9.78 \\
\hline & $09 \mathrm{Qz}$ & $q F S-c h r 24-1$ & 24 & NAU4064 & CGR6508 & 99.98 & 3.03 & -0.20 & -0.67 & 9.51 \\
\hline \multirow[t]{11}{*}{ Fiber elongation } & $08 \mathrm{Hj}$ & $q F E-\operatorname{ch} r 5-1 * *$ & 5 & HAU746 & HAU1315 & 39.76 & 4.95 & -0.05 & -0.01 & 10.62 \\
\hline & $09 \mathrm{Qz}$ & & 5 & HAU911 & HAU746 & 39.08 & 4.66 & -0.05 & 0.01 & 10.55 \\
\hline & $08 \mathrm{Hj}$ & $q F E-\operatorname{chr} 10-1 *$ & 10 & BNL2960 & CGR5873 & 65.66 & 2.87 & -0.05 & 0.02 & 5.89 \\
\hline & $09 \mathrm{Qz}$ & & 10 & NAU3404 & GH199 & 64.97 & 3.54 & 0.01 & 0.04 & 7.67 \\
\hline & 07Hn & $q F E-\operatorname{chr} 24-1$ *\# $^{\#}$ & 24 & DPL588 & GH197 & 132.22 & 3.70 & -0.02 & 0.09 & 10.76 \\
\hline & $08 \mathrm{Hj}$ & & 24 & DPL588 & GH197 & 132.22 & 3.73 & -0.04 & -0.01 & 8.06 \\
\hline & $09 \mathrm{Qz}$ & & 24 & CGR6508 & DPL588 & 119.15 & 2.85 & -0.02 & -0.02 & 9.44 \\
\hline & 07Hn & $q F E-c h r 4-1$ & 4 & BNL1167 & HAU1332 & 32.79 & 3.31 & -0.07 & 0.07 & 9.28 \\
\hline & $08 \mathrm{Hj}$ & $q F E-\operatorname{chr} 10-2^{\#}$ & 10 & CGR5873 & GH144 & 89.43 & 5.01 & 0.05 & 0.02 & 13.70 \\
\hline & $08 \mathrm{Qz}$ & $q F E-c h r 24-2$ & 24 & NAU3934 & CGR5423 & 56.54 & 4.79 & 0.00 & 0.05 & 10.84 \\
\hline & $08 \mathrm{Qz}$ & $q F E-c h r 25-1$ & 25 & CER042 & GH220 & 35.92 & 3.82 & -0.01 & -0.03 & 9.02 \\
\hline \multirow[t]{7}{*}{ Fiber micronaire } & $08 \mathrm{Hj}$ & $q F M-\operatorname{chr} 19-1 *$ & 19 & CGR5539 & NAU2894 & 67.87 & 3.29 & 0.17 & 0.00 & 9.66 \\
\hline & 09Qz & & 19 & CGR5539 & NAU2894 & 65.87 & 2.34 & 0.01 & 0.19 & 9.59 \\
\hline & $08 \mathrm{Qz}$ & $q F M-c h r 3-1$ & 3 & CGR6528 & CGR6017 & 39.95 & 3.05 & -0.17 & 0.14 & 6.48 \\
\hline & 08Qz & $q F M$-chr $9-1^{\#}$ & 9 & DC40407 & NAU5474 & 65.01 & 4.59 & -0.27 & 0.04 & 13.16 \\
\hline & $08 \mathrm{Hj}$ & $q F M$-chr $9-2^{\#}$ & 9 & DC40129a & GH247 & 131.23 & 3.06 & -0.02 & -0.23 & 13.27 \\
\hline & $09 \mathrm{Qz}$ & $q F M-c h r 19-2$ & 19 & DPL210 & GH72 & 37.45 & 4.72 & -0.17 & 0.07 & 11.13 \\
\hline & $08 \mathrm{Hj}$ & $q F M-\operatorname{chr} 26-1$ & 26 & NAU2175 & DPL491 & 44.01 & 3.27 & 0.15 & 0.00 & 16.02 \\
\hline
\end{tabular}

a) Additive effect: positive values indicate that GX1135 alleles increase the traits scores, and negative values indicate that GX100-2 alleles increase the trait scores; b) dominance effect: positive values of the dominance effect indicate that heterozygotes have higher phenotypic values than the respective means of two homozygotes, and negative values indicate that heterozygotes have lower values than the means of the two homozygotes; c) phenotypic variation explained by a single QTL. \#, The QTL was detected in combined analysis. *, QTL were identified in two or more environments.

(i) Fiber length. Twelve QTLs for fiber length were identified, in which QTLs $q F L$-chr5-2 and qFL-chr14-2 were detected in the four environments and in the combined analysis simultaneously (Table 4, Figure 1). QTL $q F L$-chr5-2 was mapped in the same interval in the four environments but gene effects differed in magnitude, which had a range of phenotypic variance (PV) from $5.40 \%$ to $17.43 \%$. At this QTL, the GX100-2 allele increased fiber length. QTL $q F L$ chr14-2 was another major QTL detected in the four environments. All additive effects were positive, indicating that the GX1135 allele increased phenotypic effect. The two QTLs should be suitable for MAS in cotton breeding because they were stably expressed in all experimental environments.

(ii) Fiber uniformity ratio. Five QTLs for fiber uniformity ratio were identified on chromosomes $2,5,14,18$, and 26 (Table 4). Two QTLs were identified on chromosomes 2 and 5 in more than one environment. The additive effect of QTL $q F U$-chr2-1 was negative in the 07Hn environment, indicating that the GX100-2 allele increases phenotypic effect, whereas the additive effect was positive in the $08 \mathrm{Hj}$ environment, indicating that the GX1135 allele increases phenotypic effect; this QTL contributed 7.35\% and $6.09 \%$ of phenotypic effect in $07 \mathrm{Hn}$ and $08 \mathrm{Hjv}$ environments, respectively. QTL $q F U$-chr $5-1$ contributed $11.42 \%$ and 
chr1

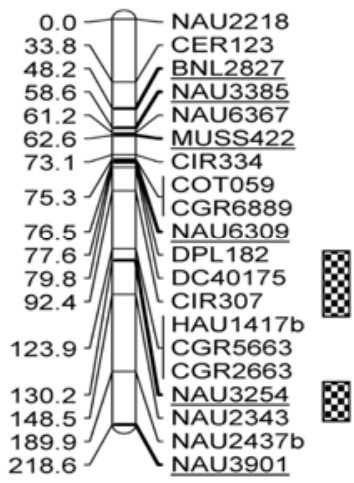

chr3

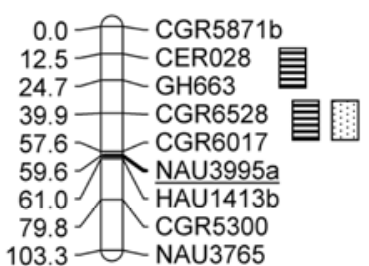

chr9

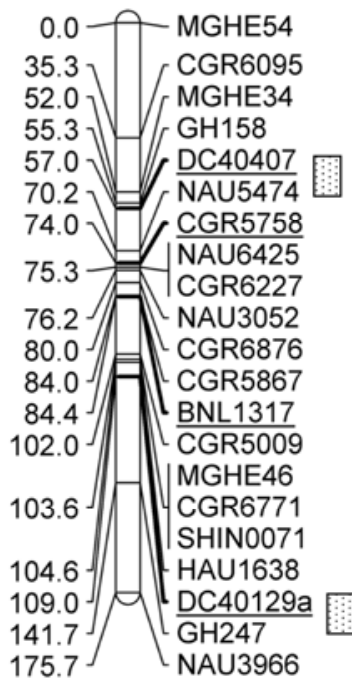

chr10

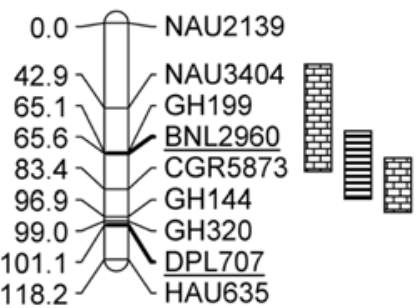

chr2
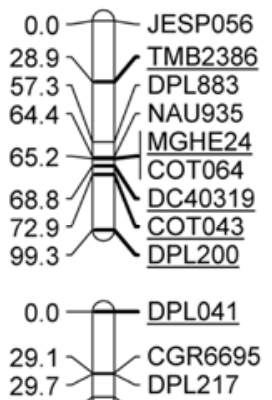

DPL217
SHIN0029

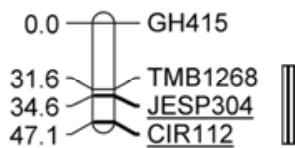

0.0 NAU2265

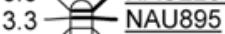

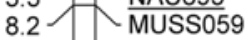

$39.0-\underline{\text { NAU } 2277}$

chr4

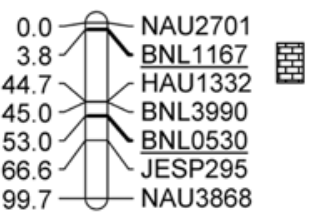

chr11
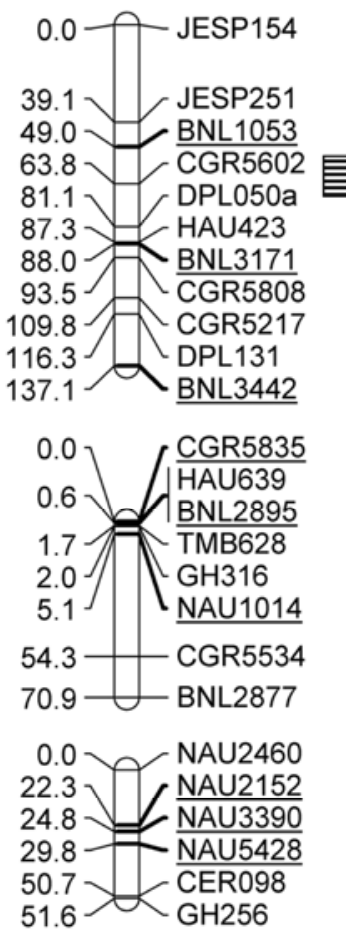

chr5
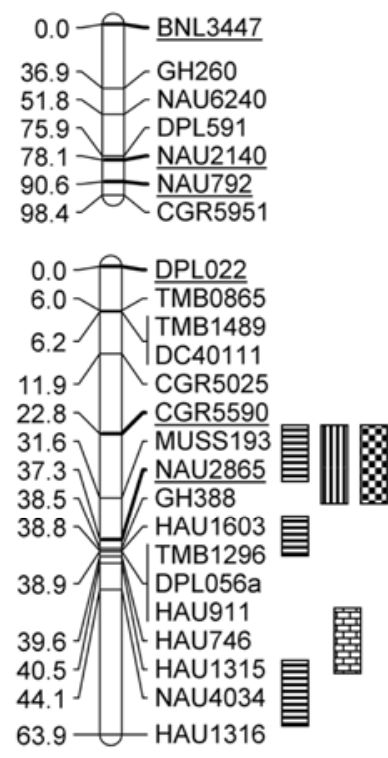

chr8

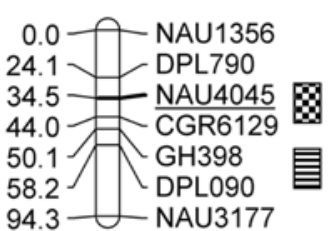

chr12
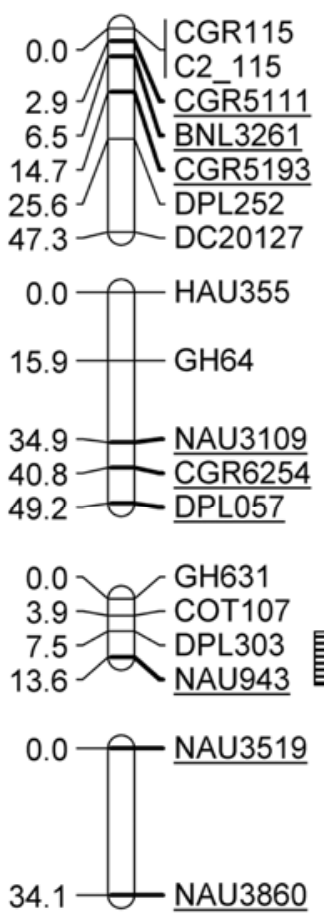
chr13

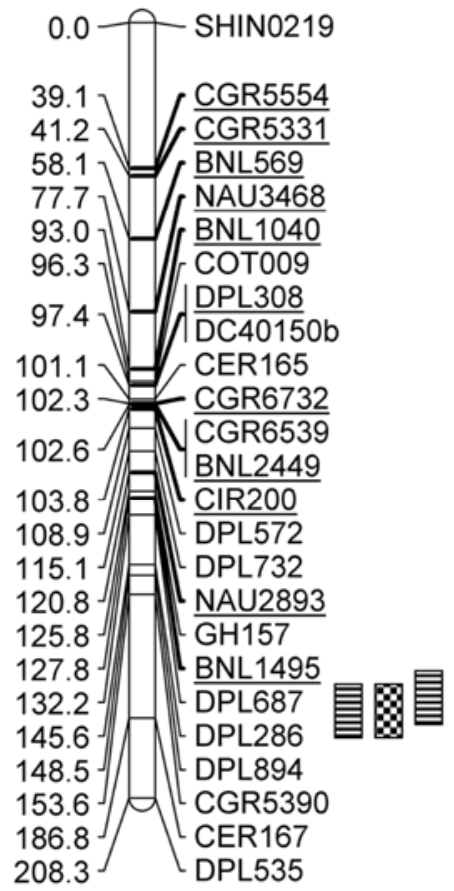

chr18
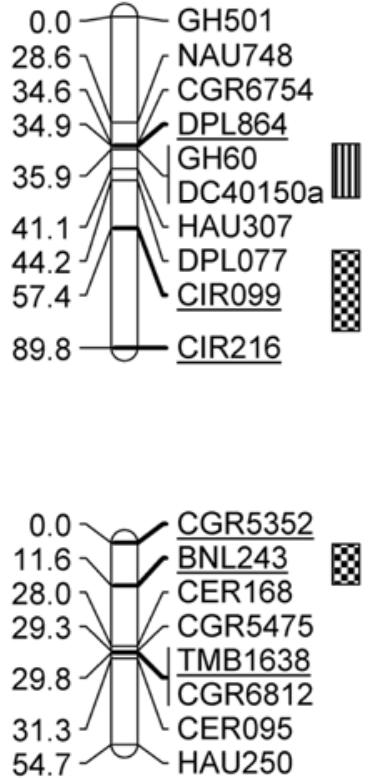

chr14

chr17
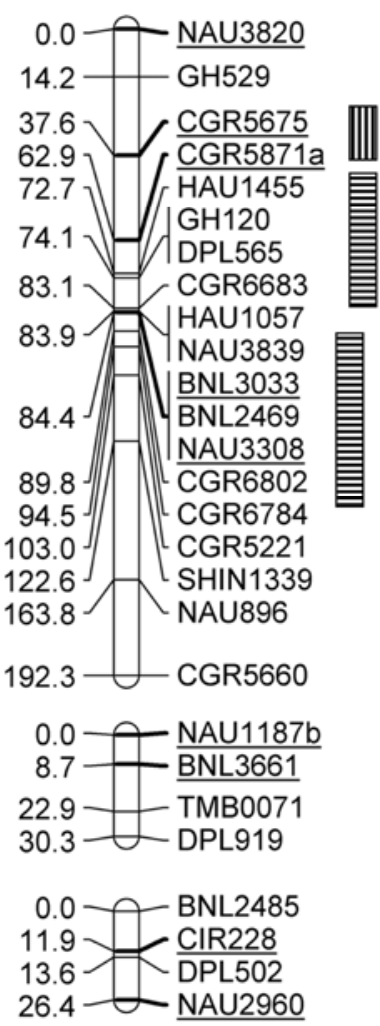

chr19
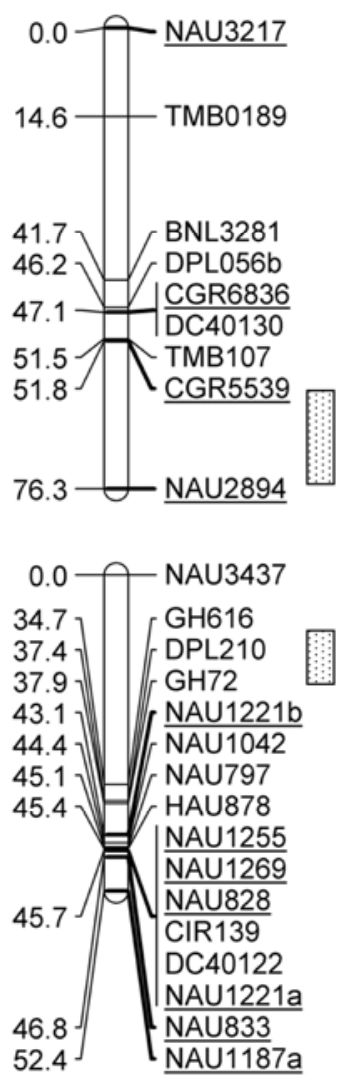

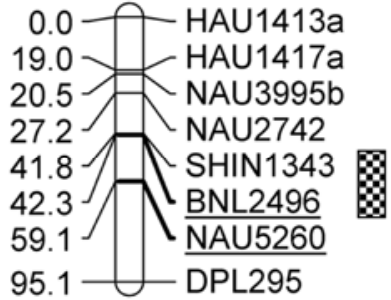

chr25
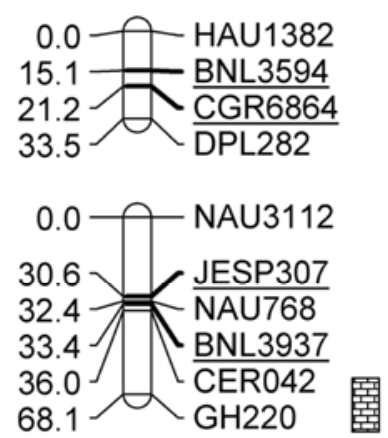

chr24
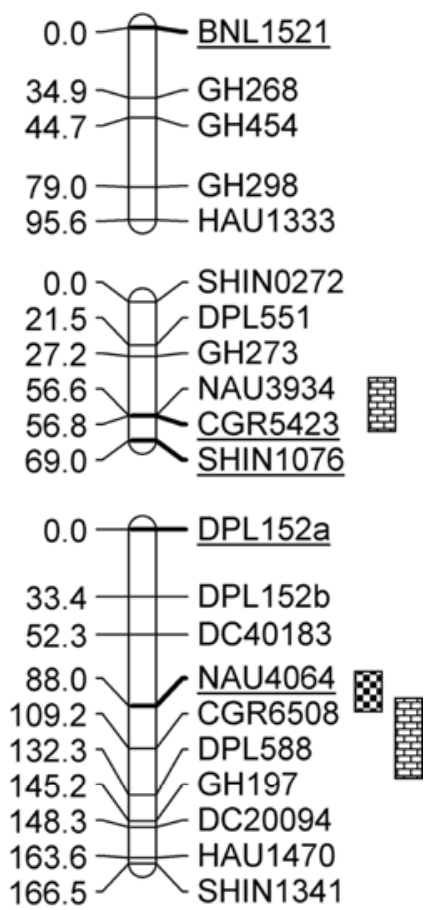

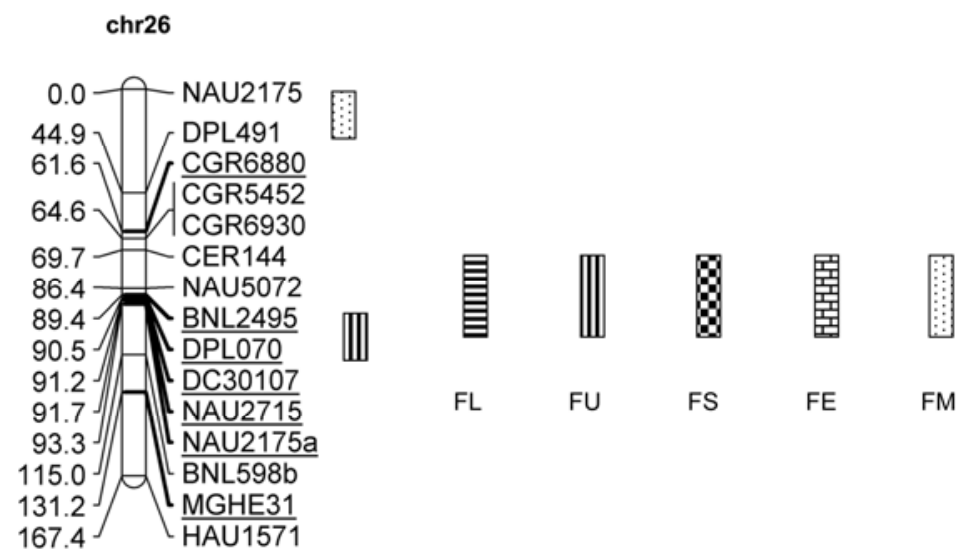

Figure 1 Mapping of QTLs for fiber quality traits in four environments. FL, fiber length; FU, fiber uniformity; FM, fiber micronaire; FE, fiber elongation; FS, fiber strength (Only those chromosomes which QTL were mapped to are shown). Markers underlined were published previously.

$15.93 \%$ of $\mathrm{PV}$ in the $08 \mathrm{Qz}$ and $08 \mathrm{Hj}$ environments, respectively. This QTL were also detected in the combined analysis.

(iii) Fiber strength. Nine QTLs for fiber strength were detected in which two QTLs, $q F S$-chr5-1 and $q F S$-chr18-1, were detected in the combined analysis simultaneously (Table 4). QTL $q F S$-chrl-1 was detected in three environments, explaining from $7.06 \%$ to $12.46 \%$ of $\mathrm{PV}$, and its additive effect was negative, indicating that the GX100-2 allele increases PV. QTL $q F S$-chr5-1 was also detected in three environments. The QTLs $q F L-c h r 5-1$ and $q F U$-chr5-1 were also identified and mapped in the same interval, suggesting that these traits are controlled by the same genes and represent QTLs with pleiotropic effects.

(iv) Fiber elongation. Seven significant QTLs for fiber elongation were detected on chromosomes 4, 5, 10, 24, and 25 (Table 4). QTL $q F E$-chr24-1 was identified and mapped in neighboring intervals in the three environments, and which was detected in the combined analysis too, with a range of LOD scores from 2.85 to 3.73 . At this QTL, the GX1135 allele conferred finer fiber. In addition, a significant QTL qFE-chr10-2 was meanwhile detected on chromosome 10 in the combined analysis, which showed the largest effects and explained $13.70 \%$ of PV.

(v) Fiber micronaire. Six QTLs for fiber micronaire were identified. QTL $q F M$-chr19-1 was identified in two environments. Its additive effect was positive, indicating that the GX1135 allele increases PV, contributing 9.66\% and $9.59 \%$ of $\mathrm{PV}$ in $08 \mathrm{Hj}$ and $09 \mathrm{Qz}$ environments, respectively. In addition, QTL $q F M-c h r 9-1$ and $q F M-c h r 9-2$ were detected in the combined analysis, which explained $13.16 \%$ and $13.27 \%$ of PV, respectively (Table 4).

\section{Discussion}

\subsection{QTLs for cotton fiber quality traits}

Here we found many common characteristics of QTLs related to fiber quality traits as described in the previous re- ports involving interspecific maps [3,10,23,33-38] and intraspecific maps [12,15,26,30,39-46], although few common markers were used in the present research and the previous studies, and the maps covered different region parts of cotton genome, making it difficult to compare the common QTLs, some QTLs were detected and mapped on the same chromosomes and affect common traits. These include seven QTLs for fiber length located in the same chromosomal regions as reported earlier [10,26,34,41,42], two QTLs for fiber uniformity ratio located in the same chromosomal regions [43,46], two QTLs for fiber micronaire [10,43,44], three QTLs for fiber elongation [30,36,42,43,46,47], and 4 QTLs for fiber strength [26,34,36,43,44,46,47]. For example, the chromosome regions where QTLs were detected for fiber length in our research matched those in an interspecific map developed from an $F_{2}$ population [10]; the QTLs may be common QTLs for fiber quality traits. These stable and consensus QTLs for fiber quality traits will enable the use of MAS to improve fiber quality of future cotton cultivars. To use MAS and dissect the genetic basis of fiber quality traits, however, it is necessary to develop more common SSR markers for use by different research groups.

\subsection{Marker assisted selection for fiber quality traits}

The demand for improved cotton fiber quality has risen with the advent of open-end, air-jet, and vortex spinning. Breeders have long recognized a significant negative association between lint yield and fiber quality. Although conventional breeding has played a vital role in the genetic improvement of lint yield and fiber quality in upland cotton, the achievement and progress has been slow [14].

The utilization of molecular markers makes it possible for plant breeders to identify rapid and precise approaches to conventional selection schemes [7]. For example, the major QTL QTLFS1 was detected in three environments (Nanjing and Hainan, China, and College Station, Texas, USA) using $\mathrm{F}_{2}$ and $\mathrm{F}_{3}$ populations derived from a cross be- 
tween 7235 and TM-1; this QTL was found to be associated with eight markers and explained more than $30 \%$ of the PV [45]. MAS revealed that DNA markers linked to QTLFS1 could be used to increase the fiber strength of upland cotton. Guo et al. [48] provided a successful example of MAS pyramiding for QTLs for favorable traits in breeding programs; two QTLs were detected for fiber strength, which greatly improved the selection efficiency for fiber strength by MAS. Shi et al. [49] used two SSR markers linked tightly with a major QTL for fiber strength to increase fiber strength through MAS. Dong et al. [50] pyramided high fiber strength genes and an insect resistance gene by MAS. The results indicated that the genetic effect of the QTL was stable and significant in different environmental conditions. Thus, major QTLs were both possible and efficient to improve fiber quality of cotton by MAS. In the $t 1$ locus region on chromosome 6, Wan et al. [51] detected QTLs affecting fiber length, fiber strength, fiber length uniformity, and spiny bollworm resistance that increased the trait phenotypic values. Because all the QTLs were mapped within about $5 \mathrm{cM}$ of the $t 1$ locus, this locus could be considered as the candidate gene for the QTLs, which should be particularly useful in MAS manipulation of fiber yield and quality.

In the present research, QTLs $q F L-c h r 5-2$ and $q F L$ chrl4-2 for fiber length were detected in three generations (four environments) and the combined analysis (data from means of different environments in $\mathrm{F}_{2: 3}$ generation) simultaneously. That is, the two QTLs were expressed stably in different generations and environments, which should be useful for MAS for fiber quality traits in upland cotton. Dong et al. [51] screened three SSR markers linked with QTLs for fiber length and studied the effect of MAS and pyramiding breeding in the three combinations. These results suggest that the construction of a saturated linkage map for G. hirsutum, the DNA markers associated with the QTL is an effective means to improve fiber quality by MAS and by pyramiding QTL in upland cotton breeding programs.

This work was supported by a grant from the National High Technology Research and Development Program (2011AA10A102), in part by the National Natural Science Foundation of China (31171591) and a grant from the New Century Excellent Talents of the Ministry of Education (NCET-06-0106) to J HUA.

1 Percival A, Wendel J, Stewart J. Taxonomy and germplasm resourses. In: Smith C W, Cothren J T, eds. Cotton: Origon, History, Technology, and Production. New York: Wiley, 1999. 33-63

2 Chen Z J, Scheffler B E, Dennis E, et al. Toward sequencing cotton (Gossypium) genomes. Plant Physiol, 2007, 145: 1303-1310

3 Kohel R J, Yu J, Park $\mathrm{Y} \mathrm{H}$, et al. Molecular mapping and characterization of traits controlling fiber quality in cotton. Euphytica, 2001, 121: 163-172

4 Shen X, Guo W, Lu Q, et al. Genetic mapping of quantitative trait loci for fiber quality and yield trait by RIL approach in upland cotton. Euphytica, 2007, 155: 371-380

5 Zhang Z S, Li X B, Xiao Y H, et al. Combining ability and heterosis between high strength lines and transgenic Bt (Bacillus thuringiensis) bollworm-resistant lines in upland cotton (Gossypium hirsutum L.). Agricult Sci China, 2003, 2: 13-18

6 Chen X, Guo W, Liu B, et al. Molecular mechanisms of fiber differential development between $G$. barbadense and $G$. hirsutum revealed by genetical genomics. PLoS One, 2012, 7: e30056

7 Tanksley S, Hewitt J. Use of molecular markers in breeding for soluble solids content in tomato: A re-examination. Theor Appl Genet, 1988, 75: 811-823

8 Guo W, Cai C, Wang C, et al. A microsatellite-based, gene-rich linkage map reveals genome structure, function and evolution in Gossypium. Genetics, 2007, 176: 527-541

9 Guo W, Cai C, Wang C, et al. A preliminary analysis of genome structure and composition in Gossypium hirsutum. BMC Genomics, 2008, 9: 314-332

10 He D H, Lin Z X, Zhang X L, et al. QTL mapping for economic traits based on a dense genetic map of cotton with PCR-based markers using the interspecific cross of Gossypium hirsutum $\times$ Gossypium barbadense. Euphytica, 2007, 153: 181-197

11 Yu Y, Yuan D, Liang S, et al. Genome structure of cotton revealed by a genome-wide SSR genetic map constructed from a BC1 population between Gossypium hirsutum and G. barbadense. BMC Genomics, 2011, 12: 15

12 Lin Z, Zhang Y, Zhang X, et al. A high-density integrative linkage map for Gossypium hirsutum. Euphytica, 2009, 166: 35-45

13 Wang B, Guo W, Zhu X, et al. QTL mapping of yield and yield components for elite hybrid derived-RILs in upland cotton. J Genet Genom, 2007, 34: 35-45

14 Zhang Z S, Hu M C, Zhang J, et al. Construction of a comprehensive PCR-based marker linkage map and QTL mapping for fiber quality traits in upland cotton (Gossypium hirsutum L.). Mol Breed, 2009, 24: 49-61

15 Zhang K, Zhang J, Ma J, et al. Genetic mapping and quantitative trait locus analysis of fiber quality traits using a three-parent composite population in upland cotton (Gossypium hirsutum L.). Mol Breed, 2012, 29: 335-348

16 Lin L, Pierce G, Bowers J, et al. A draft physical map of a D-genome cotton species (Gossypium raimondii). BMC Genomics, 2010, 11: 395-412

17 Wang K, Wang $\mathrm{Z}$, Li F, et al. The draft genome of a diploid cotton Gossypium raimondii. Nat Genet, 2012, 44: 1098-1103

18 Paterson A H, Wendel J F, Gundlach H, et al. Repeated polyploidization of Gossypium genomes and the evolution of spinnable cotton fibres. Nature, 2012, 492: 423-427

19 Hoagland D, Arnon D. The water-culture method for growing plants without soil. Circular No. 347, California Agricultural Experiment Station, 1950

20 Paterson A, Brubaker C, Wendel J. A rapid method for extraction of cotton (Gossypium spp.) genomic DNA suitable for RFLP or PCR analysis. Plant Mol Biol Rep, 1993, 11: 122-127

21 Lacape J, Nguyen T, Thibivilliers S, et al. A combined RFLPCSSR-CAFLP map of tetraploid cotton based on a Gossypium hirsutum $\times$ Gossypium barbadense backcross population. Genome, 2003, 46: 612-626

22 Rong J, Abbey C, Bowers J, et al. A 3347-locus genetic recombination map of sequence-tagged sites reveals features of genome organization, transmission and evolution of cotton (Gossypium). Genetics, 2004, 166: $389-417$

23 Xiao J, Wu K, Fang D, et al. New SSR markers for use in cotton (Gossypium spp.) improvement. J Cotton Sci, 2009, 13: 75-157

24 Mei M, Syed N, Gao W, et al. Genetic mapping and QTL analysis of fiber-related traits in cotton (Gossypium). Theor Appl Genet, 2004, 108: 280-291

25 Lander E S, Green P, Abrahamson J, et al. MAPMAKER: An interactive computer package for constructing primary genetic linkage maps of experimental and natural populations. Genomics, 1987, 1: 174-181

26 Qin H, Guo W, Zhang Y, et al. QTL mapping of yield and fiber traits based on a four-way cross population in Gossypium hirsutum L. Theor Appl Genet, 2008, 117: 883-894

27 Zeng Z. Precision mapping of quantitative trait loci. Genetics, 1994, 
136: $1457-1468$

28 Wang S, Basten C, Zeng Z. Windows QTL cartographer 2.5. Department of Statistics, North Carolina State University, Raleigh, 2005

29 Lander E, Kruglyak L. Genetic dissection of complex traits: Guidelines for interpreting and reporting linkage results. Nat Genet, 1995, 11: 241-247

30 Shen X, Guo W, Zhu X, et al. Molecular mapping of QTLs for fiber qualities in three diverse lines in upland cotton using SSR markers. Mol Breed, 2005, 15: 169-181

31 Zhang Y M, Xu S Z. Mapping quantitative trait loci in $F_{2}$ incorporating phenotypes of $F_{3}$ progeny. Genetics, 2004, 166: 1981-1993

32 Voorrips R. MapChart: Software for the graphical presentation of linkage maps and QTLs. J Hered, 2002, 93: 77-78

33 Jiang C X, Wright R J, El-Zik K M, et al. Polyploid formation created unique avenues for response to selection in Gossypium (cotton). Proc Natl Acad Sci USA, 1998, 95: 4419-4424

34 Lacape J M, Nguyen T B, Courtois B, et al. QTL analysis of cotton fiber quality using multiple Gossypium hirsutum $\times$ Gossypium barbadense backcross generations. Crop Sci, 2005, 45: 123-140

35 Lacape J M, Jacobs J, Arioli T, et al. A new interspecific, Gossypium hirsutum $\times$ barbadense, RIL population: Towards a unified consensus linkage map of tetraploid cotton. Theor Appl Genet, 2009, 119: 281-292

36 Paterson A, Saranga Y, Menz M, et al. QTL analysis of genotypex environment interactions affecting cotton fiber quality. Theor Appl Genet, 2003, 106: 384-396

37 Reinisch A J, Dong J M, Brubaker C L, et al. A detailed RFLP map of cotton, Gossypium hirsutum $\times$ Gossypium barbadense: Chromosome organization and evolution in a disomic polyploid genome. Genetics, 1994, 138: 829-847

38 Yu J, Park Y, Lazo G, et al. Molecular mapping of the cotton genome: QTL analysis of fiber quality properties. Beltwide Cotton Conferences (USA), 1998, 1: 485

39 Chen H, Qian N, Guo W, et al. Using three overlapped RILs to dissect genetically clustered QTL for fiber strength on Chro. D8 in upland cotton. Theor Appl Genet, 2009, 119: 605-612

40 Shappley Z, Jenkins J N, Zhu J, et al. Quantitative trait loci associated with agronomic and fiber traits of upland cotton. J Cotton Sci, 1998, 2: 153-163

41 Ulloa M, Meredith Jr W R. Genetic linkage map and QTL analysis of agronomic and fiber quality traits in an intraspecific population. $\mathrm{J}$ Cotton Sci, 2000, 4: 161-170

42 Wang B, Guo W, Zhu X, et al. QTL mapping of fiber quality in an elite hybrid derived-RIL population of upland cotton. Euphytica, 2006, 152: 367-378

43 Wang B, Wu Y, Guo W, et al. QTL analysis and epistasis effects dissection of fiber qualities in an elite cotton hybrid grown in second generation. Crop Sci, 2007, 47: 1384-1392

44 Wu J, Gutierrez O A, Jenkins J N, et al. Quantitative analysis and QTL mapping for agronomic and fiber traits in an RI population of upland cotton. Euphytica, 2009, 165: 231-245

45 Zhang T, Yuan Y, Yu J, et al. Molecular tagging of a major QTL for fiber strength in upland cotton and its marker-assisted selection. Theor Appl Genet, 2003, 106: 262-268

46 Zhang Z S, Xiao Y H, Luo M, et al. Construction of a genetic linkage map and QTL analysis of fiber-related traits in upland cotton (Gossypium hirsutum L.). Euphytica, 2005, 144: 91-99

47 Chee P, Draye X, Jiang C X, et al. Molecular dissection of interspecific variation between Gossypium hirsutum and Gossypium barbadense (cotton) by a backcross-self approach. I. Fiber elongation. Theor Appl Genet, 2005, 111: 757-763

48 Guo W, Zhang T, Ding Y, et al. Molecular marker assisted selection and pyramiding of two QTLs for fiber strength in upland cotton. Acta Genet Sin, 2005, 32: 1275-1285

49 Shi Y, Liu A, Li J. The major QTLs linked to fiber strength for cotton breeding program by molecular marker assisted selection. Mol Plant Breed, 2007, 5: 521-527

50 Dong Z, Shi Y, Zhang J, et al. Molecular marker-assisted selection and pyramiding breeding of major QTLs for cotton fiber length. Cotton Sci, 2009, doi: CNKI: SUN: MHXB.0.2009-04-007

51 Wan Q, Zhang Z, Hu M, et al. T1 locus in cotton is the candidate gene affecting lint percentage, fiber quality and spiny bollworm (Earias spp.) resistance. Euphytica, 2007, 158: 241-247

Open Access This article is distributed under the terms of the Creative Commons Attribution License which permits any use, distribution, and reproduction in any medium, provided the original author(s) and source are credited.

\section{Supporting Information}

Figure S1 Variation of fiber quality traits in $\mathrm{F}_{2}, \mathrm{~F}_{2: 3}$ and $\mathrm{F}_{2: 4}$ populations.

The supporting information is available online at csb.scichina.com and www.springerlink.com. The supporting materials are published as submitted, without typesetting or editing. The responsibility for scientific accuracy and content remains entirely with the authors. 